\title{
Is radioiodine treatment for thyroid cancer a risk factor for second primary malignancies?
}

\author{
Massimo Eugenio Dottorini - Massimo Salvatori
}

Received: 21 February 2013/Accepted: 13 May 2013/Published online: 23 May 2013

(C) Italian Association of Nuclear Medicine and Molecular Imaging 2013

\begin{abstract}
Radioiodine is a safe and well-established therapeutic modality for the ablation of thyroid remnants and the treatment of locoregional and distant metastases from differentiated thyroid carcinoma. However, a careful hazard assessment is mandatory in order to establish the risk-benefit ratio, especially in low-risk patients. Induction of second primary malignancies is one of the most serious possible untoward effects of radioiodine treatment. The limited incidence both of this effect and of thyroid cancer and the paucity of available dosimetric data make it difficult to perform high-quality studies that could provide evidence-based indications. A number of bias and confounding factors can blur data and must be considered, evaluating studies, addressing this topic. Data from the main surveys confirm that radioiodine can induce second primary tumors, with a probability comparable to that of external radiotherapy and lower than that of chemotherapy. Even though radioiodine treatment of thyroid cancer carries a low relative risk, the process of therapy justification must be carefully conducted and every measure that can reduce patient exposure must be taken, especially in children, adolescents and young adults.
\end{abstract}

Keywords Thyroid neoplasms - Iodine radioisotopes/ adverse effects - Iodine radioisotopes/therapeutic use . Neoplasms · Radiation-induced/etiology $\cdot$ Second primary/ etiology

M. E. Dottorini ( $\square)$

S.C. Medicina Nucleare, "S. Maria della Misericordia" Hospital,

Sant'Andrea delle Fratte, 06156 Perugia, Italy

e-mail: massimo.dottorini@ospedale.perugia.it

M. Salvatori

Istituto di Medicina Nucleare, Università Cattolica del Sacro

Cuore, Rome, Italy

\section{Introduction}

Differentiated thyroid carcinoma (DTC) is the most common endocrine malignancy, whose incidence is, in several countries, reported to have risen over recent decades $[1,2]$. In the United States, its incidence nearly tripled from 1973 through 2002 (from 2.7 to 7.7 cases per 100,000) and a similar increasing trend has been reported in Europe [2]. These data correspond to more than 2,100 and 48,000 cases each year in the UK and USA, respectively [3].

The protocol for the treatment of patients with thyroid carcinoma involves surgical removal of the thyroid and, in most cases, radioiodine treatment (RIT) to ablate thyroid remnants or treat residual tumor. Further therapies can be prescribed, using fixed or dosimetry-based activities of ${ }^{131} \mathrm{I}$, to treat locoregional or distant functioning metastases [4].

Whilst all current guidelines recommend the use of RIT in high-risk patients, its use for remnant ablation in lowrisk patients is still a debated question, on account of uncertainty over its benefits $[5,6]$. A randomized non-inferiority phase II/III multicenter trial to determine whether 5-year disease-free survival is any worse in patients not undergoing compared with those undergoing ablation has recently been planned in the UK (NCT01398085) [3].

Despite this uncertainty, RIT is being used more and more across all tumor sizes and in different situations, including favorable low-risk cancers in young patients with a long life expectancy, who risk being over-treated. In this subgroup of patients, the possible small reduction of recurrence obtained through RIT must be weighed up against the long-term risks [7]. While, in most cases, the early deterministic effects of radiation exposure are of scarce clinical relevance, carcinogenesis is, nevertheless, a possible and very concerning long-term complication. 
Given the discrepant results reported, the risk of second primary malignancies (SPMs) and leukemia remains a controversial topic that needs to be better understood [8].

This review looks at the issue of the possible induction of SPMs after RIT in patients with DTC. In the first section, we summarize the risks of RIT, particularly with regard to the stochastic or probabilistic effects. In the following sections, we review the molecular mechanisms of carcinogenesis induced by ionizing radiation, the carcinogenic risk in RIT of DTC, and finally the available epidemiological studies on SPMs in patients with DTC, treated or not treated with radioiodine. A glossary of the most important terms used in epidemiological studies on radiation-induced tumors is also provided ("Appendix").

\section{The risks of ${ }^{131}$ I therapy in patients with differentiated thyroid cancer}

Since radioiodine has no pharmacological effects, the hazards of RIT are all related to the exposure to ionizing radiation. The untoward effects of RIT can be categorized in different ways: by the timing of their occurrence after therapy (early or immediate, intermediate and late), by their occurrence in a "typical patient" versus a patient with high-risk factors for complications, and on the basis of the nature of the radiobiological effects, which can be classified as deterministic or stochastic [9] (Table 1).

Deterministic effects, which are related to cell killing, usually occur early, and a substantial dose of radiation is

Table 1 Characteristics of deterministic and stochastic effects

\begin{tabular}{|c|c|}
\hline Deterministic effects & Stochastic effects \\
\hline $\begin{array}{l}\text { - Severity of the effect increases } \\
\text { with the absorbed dose }\end{array}$ & $\begin{array}{l}\text { - Severity of the effect is } \\
\text { independent of absorbed dose }\end{array}$ \\
\hline $\begin{array}{l}\text { - Probability of occurrence } \\
\text { increases with dose }\end{array}$ & $\begin{array}{l}\text { - Probability of occurrence } \\
\text { increases with dose }\end{array}$ \\
\hline - Non-linear dose response & $\begin{array}{l}\text { - Linear, linear-quadratic and } \\
\text { possibly non-linear dose } \\
\text { response }\end{array}$ \\
\hline - Practical threshold dose & - No threshold dose \\
\hline $\begin{array}{l}\text { - Usually occur after high-dose } \\
\text { acute exposure }\end{array}$ & - Can also occur after low doses \\
\hline $\begin{array}{l}\text { Mechanisms: killing of large } \\
\text { groups of cells in the tissue } \\
\text { involved. Different effects, } \\
\text { tissues and individuals have } \\
\text { various threshold doses. }\end{array}$ & - Mechanisms: changes in genes \\
\hline $\begin{array}{l}\text { - Most arise within days or weeks, } \\
\text { but some can show up over } \\
\text { longer periods (years). }\end{array}$ & - Latency period of years \\
\hline $\begin{array}{l}\text { - Example: sialadenitis after }{ }^{131} \mathrm{I} \\
\text { therapy }\end{array}$ & $\begin{array}{l}\text { - Example: radiation-induced } \\
\text { cancer }\end{array}$ \\
\hline
\end{tabular}

required in order to produce them. In most cases, deterministic effects do not occur below a threshold level of absorbed radiation dose because their appearance requires depletion of certain cells in the body to below a critical number in a given organ or tissue. Above threshold, the severity of the effect increases as a function of dose. Specific health effects are typically associated with specific threshold dose levels, although they may also depend, to a limited extent, on the exposed individual. In RIT of DTC many factors affect the frequency and severity of deterministic side effects, including single and total cumulative prescribed ${ }^{131} \mathrm{I}$ activities, the time between therapies, measures implemented or not implemented to prevent the various side effects, and the presence of risk factors for side effects [9].

The main deterministic effects of RIT in patients with DTC tend to involve the salivary glands (taste disturbances, swelling, sialadenitis, xerostomia, salivary duct obstruction), eyes and nasolacrimal glands (xerophthalmia, epiphora, conjunctivitis), genital system (hypospermia and azoospermia in males and temporary amenorrhea or oligomenorrhea in females), lungs (very rare acute radiation pneumonitis and fibrosis) and bone marrow (neutropenia, low platelet count and anemia). Most of these effects are early and transient, but some may become permanent; some do not appear until months or years after treatment, such as bone marrow depression, radiation pulmonary fibrosis, early onset of menopause and chronic dry eye [9].

Unlike deterministic effects, stochastic effects do not have a threshold, occur in an arbitrary or probabilistic manner, have a severity that does not depend on dose, and occur months or years after high-level and possibly also after low-level radiation exposure. Carcinogenesis and heritable effects are considered the only stochastic events due to ionizing radiation. Induction of SPMs (solid tumors and leukemia) is considered to be the main stochastic effect of RIT in patients with DTC, the somatic effects of radiation exposure being much more probable (total somatic risk $9.5-15 / 100$ Gy) with respect to the genetic heritable effects (total heritable genetic risk $0.30-0.47 / 100$ Gy for the first generation; $0.12-0.32$ for the second generation) $[10,11]$.

Most international organizations involved in the assessment of radiation risk have postulated, on the basis of experimental and theoretical evidence and mainly for radioprotection purposes, that no threshold exists for stochastic effects, and also that minimal exposure to radiation may induce cancer [12]. Studies in radiation biology show that "a single radiation track (resulting in the lowest exposure possible) traversing the nucleus of an appropriate target cell has a low but finite probability of damaging the cell's DNA" [12]. This damage may be difficult for the cell to repair, or it may be repaired incorrectly. The BEIR VII 


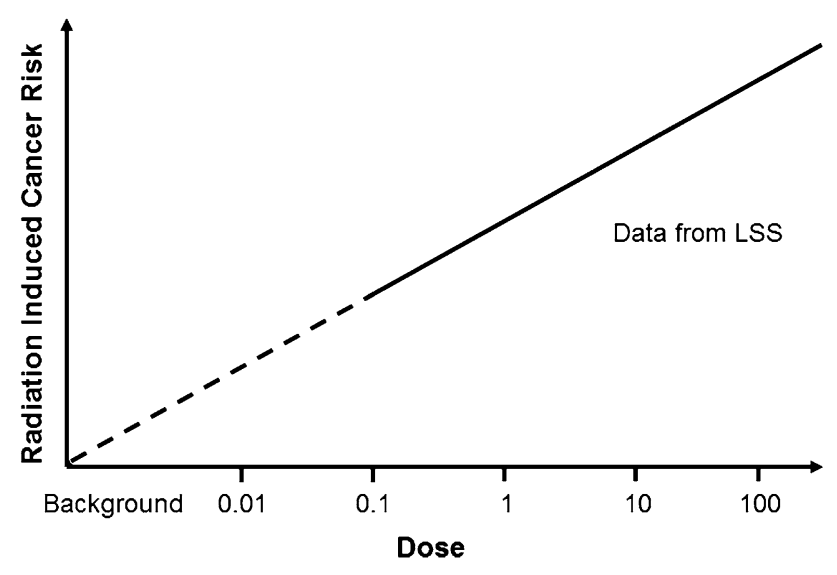

Fig. 1 Linear no-threshold (LNT) model used to estimate the radiation-induced cancer risk. LSS life span study

committee concludes that current scientific evidence is consistent with the hypothesis that there is a linear doseresponse relationship between exposure to ionizing radiation and the development of radiation-induced solid cancers in humans [13], (Fig. 1). However, although the committee deems it unlikely that a threshold exists for the induction of cancers, it notes that the occurrence of radiation-induced cancers at low doses will be small [14-16].

\section{Molecular mechanisms of carcinogenesis induced by ionizing radiation}

Radiation tumorigenesis is a complex multistage process involving intimate links between dose-related induction of DNA damage in cells, the appearance of gene or chromosomal mutations through DNA damage misrepair, and the development of cancer [13].

Error-prone repair of chemically complex DNA doublestrand damage is the predominant mechanism of radiationinduced gene or chromosomal injury involved in the carcinogenesis process, and the consequent mutations are predominantly loss-of-function DNA deletions, some of which are represented as segmental loss of chromosomal material (i.e. multigene deletions). Changes induced in genes by ionizing radiation can also produce gene mutations or DNA rearrangements, which can result in a gain of function, as in the case of conversion of proto-oncogenes to oncogenes [13].

Six biological capabilities acquired during the multistep development of human tumors are considered to be the "hallmarks of cancer" and they constitute an organizing principle for rationalizing the complexities of neoplastic disease [17]. They are the capability: (i) to sustain proliferative signaling, (ii) to evade growth suppressors, (iii) to resist cell death, (iv) to enable replicative immortality, (v) to induce angiogenesis, and (vi) to activate invasion and metastasis [17].

Radiation-induced tumors develop in a tumor-specific multistage manner that parallels that of tumors arising spontaneously. The full process may last for years before resulting in clinical disease, thus cancers attributable to radiation do not become evident until years after exposure [13]. Mutations can be related not only to radiation, but also to a wide range of environmental mutagens, or they can occur spontaneously. Because of the long latency between exposure and cancer development and the multistage nature of tumorigenesis, it is difficult to identify specific alterations induced by ionizing radiation.

Ionizing radiation produces several kinds of damage in DNA, including single-strand breaks (SSBs) and doublestrand breaks (DSBs) in DNA chains, DNA-DNA covalent cross-links, DNA-protein covalent cross-links, and a large variety of oxidative changes in the nucleotide base. The most critical defects include DSBs, which can lead to efficient cell killing or, through erroneous DNA repair, can be a source of stochastic damage, including an increased risk of malignant transformation. The general picture emerging from biophysical studies is that the misrepair of radiation-induced DNA DSBs that leads to chromosome aberrations and mutagenesis is probably associated with the dominant post-irradiation function of non-homologous end joining (NHEJ) repair processes [13].

Although chromosomal translocations can lead to oncogene activation, the main radiation-induced carcinogenic processes seem to involve a wide variety of stable chromosomal aberrations, including deletions and reciprocal translocations, rather than the point mutations predominating in spontaneous mutations. Gene and/or chromosomal rearrangements and loss of heterozygosity are thus considered the most likely radiation-induced events that contribute to cancer development [18].

Several factors may affect the dose-response relationship and have relevance for radiation-induced cancer. These include variations in radiosensitivity during the cell cycle, the induction of an adaptive response to an initial exposure, a bystander effect that causes an irradiated cell to have an effect on a nearby unirradiated cell, and novel forms of cellular damage response, collectively termed induced genomic instability [19].

The phenomenon of genomic instability has been defined as the manifestation of genetic damage (chromosomal rearrangements, chromosomal bridge formation, chromatid breaks and gaps, and micronuclei) in the progeny of somatic cells that survive irradiation [19]. Delayed lethality (i.e. reduction in cell cloning efficiency several generations after irradiation) and gene mutations that arise de novo several generations after irradiation are thought to be another manifestation of genomic instability. If such 
induced instability is involved in radiation carcinogenesis, this would imply that the initial carcinogenic event may not be a rare mutation occurring in a specific gene or set of genes. Rather, radiation may induce a process of instability in many cells in a population, enhancing the rate at which the multiple gene mutations necessary for the development of cancer may arise in a given cell lineage. Furthermore, radiation could act at any stage in the development of cancer by facilitating the accumulation of the remaining genetic events required to produce a fully malignant tumor.

In spite of major advances in understanding of the role of genetic susceptibility to radiation-induced cancer, the degree to which genetic factors may increase cancer risk remains uncertain. An important conclusion is that many cancer-prone human genetic disorders are likely to show an elevated risk of radiation-induced cancer, probably with a high degree of organ specificity. Evidence has been obtained that major deficiencies in DNA damage response and tumor-suppressor-type genes can serve to elevate cancer risk and the major practical issue associated with these findings is judged to be the risk of radiotherapyrelated cancer. Besides, major interindividual differences in radiation response seem to be due to common polymorphisms of DNA damage response genes associated with organ-wide radiation cancer risk [13].

\section{Carcinogenic risk in radioiodine therapy of differentiated thyroid cancer}

Absorbed doses to organs after administration of $1 \mathrm{GBq}$ of ${ }^{131} \mathrm{I}$ in euthyroid status to DTC patients range from a minimum of $29 \mathrm{mGy}$ for the brain and about $80 \mathrm{mGy}$ for the lungs to around $100 \mathrm{mGy}$ for the liver, spleen, kidneys and blood, and around 200 mGy for the salivary glands [20]. Such doses approach levels for which there is direct epidemiological evidence of increased cancer, as confirmed by the Biological Effects of Ionizing Radiation (BEIR) VII publication on the health risks from exposure to low levels of ionizing radiation [13].

It thus seems highly probable that RIT of DTC can have a carcinogenic effect, although it is difficult to prove this assumption with experimental data and to estimate the actual risk. Even though ionizing radiation has probably been the most intensively studied carcinogen over the last sixty years and a significant amount of knowledge has been gathered, transferring data on carcinogenic risk derived from studies on people exposed to sources and radiation types other than ${ }^{131} \mathrm{I}$ is not easy, given that cancer risk varies significantly for different types of radiation exposure [13].

As a matter of fact, the United Nations Scientific Committee on the Effects of Atomic Radiation
(UNSCEAR) evaluates separately studies on external lowLET radiation by high- and low-dose-rate exposures, internal low-LET irradiation by ${ }^{131} \mathrm{I}$, and internal high-LET radiation by radon and other exposures [21]. For example, cancer risk per unit dose for external low-LET exposures at high-dose rates tends to be higher than that at low-dose rates. Internal exposures are usually protracted and specific to certain organs and often give rise to heterogeneous radiation within organs, in contrast to most external instantaneous whole-body exposures. Finally, the relative biological effectiveness (RBE) of high-LET radiation with respect to low-LET radiation is a complex parameter that depends on the radiation type and energy, the dose and dose rate, and the endpoint under study [22].

Another caveat is that projection of cancer risk between two populations with differing underlying susceptibilities to cancer should be performed carefully. As with the projection of cancer risk over time, two models are used to transfer cancer risk across populations: in the multiplicative transfer of risks model, the ratio of the radiationinduced excess cancer rates to the underlying cancer rates is assumed to be identical in the two populations, while in the additive transfer of risks model, the radiation-induced excess cancer rates in the two populations are assumed to be identical. Depending on the cases, relative or absolute transfers of risk between populations allow a better fit of epidemiological data [22].

To prove that a certain radiation exposure is unquestionably carcinogenic, it should be shown by epidemiological studies that the probability of the effect increases with increasing radiation doses. From a practical point of view, merely assessing the carcinogenicity of RIT could be a futile exercise, whereas it would be much more useful to quantify the risk for the various levels of exposure reached in clinical management of DTC patients.

In patients with DTC treated with ${ }^{131} \mathrm{I}$, the radiation absorbed dose (expressed in Gy) may differ significantly depending not only on the cumulative administered activity (measured in GBq), but also on the uptake by various tissues of the body and on retention times in various tissues $[4,7]$. The consequent carcinogenic effect may vary, depending on cumulative administered activity, number of administrations of radioiodine, and time between each single administration. Individual internal dosimetry in RIT of DTC is a complex procedure which is performed only in selected cases and may be flawed by elevated intrinsic methodological errors [7].

Because of the relative rarity of DTC, the low risk, and the relatively low-dose levels used, epidemiological studies on the carcinogenic risk of ${ }^{131} \mathrm{I}$ therapy have to evaluate many thousands of subjects, gathered over long periods of time. Furthermore, most of the parameters that would need to be used to estimate radiation doses retrospectively are 
not provided by data extracted from cancer registries or from clinical files. Therefore, most studies on this topic only report whether or not the subject received radioiodine, and just a few investigations report cumulative administered activity, as the one parameter indirectly linked to radiation dose.

Beyond classical dosimetry, biological and physical methods could be used to estimate radiation dose [22, 23]. Although several biological samples can be considered representative of individual exposure, dose assessment is usually performed on peripheral lymphocytes. This cell population represents a suitable biological model for investigating radiation-induced effects, since it circulates in the body and remains quiescent in the G 0 stage of the cell cycle for a relatively long time. Chromosomal aberrations induced by ionizing radiation can be classified as stable or unstable anomalies. Stable anomalies, being consistent with cell survival, can persist for many years and include reciprocal, non-reciprocal, and interstitial translocations. Unstable anomalies, inducing mitotic cell death, decrease over time and include dicentrics, centric rings, and acentric aberrations. The estimate of individual exposure is obtained by comparing the dose-effect relationships for aberrations measured by scoring with the doseresponse relationships for aberrations observed in in vitro models [13].

Because of the fast disappearance of lymphocytes carrying unstable aberrations, biological dosimetry can present major limitations for long retrospective studies. For example, the method based on measurement of the phosphorylated form of the histone $\mathrm{H} 2$ variant $\mathrm{H} 2 \mathrm{AX}$ $(\gamma-\mathrm{H} 2 \mathrm{AX})$ formed over large chromatin domains surrounding DSBs as a result of exposure to ionizing radiation, despite being sensitive to doses of a few milligrays and linearly related to dose across a broad dose range, shows severe limitations, associated mainly with the rapid loss of the $\gamma-\mathrm{H} 2 \mathrm{AX}$ signal following irradiation; as a result, this method is suitable only for radiation exposure not older than two days.

Several years after exposure, scoring of stable aberrations might appear a more appropriate approach, even though it involves more laborious and expensive techniques. Some examples of biological and physical methods are classical cytogenetics for translocations, the glycophorin A mutational assay of red blood cells, the fluorescent in situ hybridization (FISH) technique for analysis of stable chromosomal translocations, and electron spin resonance (ESR), also known as electron paramagnetic resonance (EPR), of tooth enamel. Unfortunately, these methods are both complex and expensive, and cannot therefore be applied to thousands of people, while some biological measures can be affected by other factors, such as age and smoking.
In patients undergoing RIT for DTC, biological dosimetry methods have been used to estimate radiation exposure and genome damage both in adults [24] and in children and adolescents [25]. Lassmann et al. [24], in a series of twenty-six patients submitted to RIT for DTC, evaluated protracted, continuous, internal whole-body irradiation from radioiodine, using $\gamma-\mathrm{H} 2 \mathrm{AX}$ and the $\mathrm{p} 53-$ binding protein 1 (53BP1) as markers of DSBs resulting from exposure. The authors concluded that the $\gamma-\mathrm{H} 2 \mathrm{AX}$ and 53BP1 tests appear to be well suited for the detection of radiation exposure after radionuclide incorporation, even in low-dose and low-dose-rate scenarios. However, because of the high interpatient variability in ionizing radiation-induced foci enumerated by the $\gamma$-H2AX and 53BP1 tests, a reliable dose-response relationship between the absorbed dose-rate and number of induced foci was established only within individual patients rather than across a group of patients. This observation seems to limit the utility of these tests to the setting of patientspecific dosimetry.

Federico et al. [25] investigated whether administration of radioiodine may induce early genome damage in peripheral $\mathrm{T}$ lymphocytes in adolescents with DTC. The authors studied 11 youngsters treated with ${ }^{131} \mathrm{I}$ to ablate thyroid remnants; they used micronucleus assay and evaluated the expression of some genes involved in the DNA repair or apoptosis pathways. They demonstrated that peripheral cells in DTC children and adolescents who received a mean administered activity of $3.50 \pm 0.37 \mathrm{GBq}$ of ${ }^{131}$ I did not show chromosomal damage within $48 \mathrm{~h}$ of RIT administration. This may be due to prompt activation of the cell machinery that maintains the integrity of the genome to prevent harmful DSBs from progressing to chromosome mutations, either by repairing the lesions or by eliminating the most seriously damaged cells via apoptosis.

\section{Epidemiological studies on second primary malignancies in patients with differentiated thyroid cancer}

It is known that individuals with cancer are at increased risk of subsequent tumors. Because individuals diagnosed with cancer are now living longer and also because of the aging of the population, the number of people who will develop multiple malignancies is expected to increase. Mariotto et al. [26] estimated that 756,467 people in the United States were affected by cancer more than once between 1975 and 2001, and these cases represent almost $8 \%$ of the current cancer survivor population. Risk may be due to several factors, including genetic predisposition, environmental exposure, exposure to carcinogenic cancer 
therapies (i.e., iatrogenic risks associated with chemotherapy, radiotherapy and/or hormonal therapy), lifestyle factors, such as smoking, and, perhaps most importantly, aging [26]. Patients with DTC show five-and ten-year relative survival rates both close to $95 \%$, and have a high likelihood of being diagnosed with a second primary cancer in their lifetime.

The most exhaustive publication on the risk of SPMs in thyroid cancer survivors is a systematic review and meta-analysis by Subramanian et al. published in Thyroid in 2007 [27]. The authors examined thirteen full-text papers and reported pooled data from six studies of 70,844 thyroid cancer survivors. In these studies, population-based or cancer registry-based data were used as a comparator for expected incidence rates of primary cancer. The results of the systematic review and meta-analysis showed an increased incidence of SPMs in thyroid survivors with a standardized incidence ratio (SIR) of 1.20 (95\% CI 1.17-1.24), but there were insufficient data for pooling of SPM mortality data [27]. A positive significant association between thyroid cancer and risk of SPMs was found for the following tumors: salivary glands, stomach, colon/colorectal, breast, prostate, kidney, brain/central nervous system, soft sarcoma, non-Hodgkin's lymphoma, multiple myeloma, leukemia, bone/ joints, and adrenal. A significant reduced risk of lung and cervical cancers was observed.

Ronckers et al. [28] reported similar results; they found an $11 \%$ increased risk of SPMs among 29,456 survivors of thyroid cancer $(\mathrm{O} / \mathrm{E}=1.11, \mathrm{O}=2,214$, $\mathrm{EAR}=8$ per 10,000 person-years) with a cumulative incidence of developing any second cancer following thyroid cancer, adjusted for the competing risk of death from other causes, of $15.9 \%$ at 25 years $(95 \%$ $\mathrm{CI}=15.0-16.8 \%)$.

Subramanian et al. [27] and Ronckers et al. [28] evaluated the risk of SPMs in thyroid cancer survivors but did not explore the relationship between risk and thyroid cancer treatment. Although ${ }^{131} \mathrm{I}$ has been suggested to play a possible role, many other competing factors may induce an SPM in patients with thyroid cancer, such as common etiological factors, lifestyle choices, or intense medical surveillance [28]. There is increasing evidence to support the hypothesis that DTC is a polygenic disease [29] and both familial presentation of DTC [30] and familial syndromes associated with DTC and other tumors, such as Cowden syndrome [31] and familial adenomatous polyposis [32], have been described. Moreover, the reported bidirectional association (i.e., increased risk of SPMs in patients with DTC and risk of thyroid cancer developing after many other types of primary cancer) raises the possibility of shared genetic or environmental risk factors [33].
Epidemiological studies on second primary malignancies in patients with differentiated thyroid cancer treated with radioiodine

Data on the prevalence of SPMs in patients receiving RIT for DTC are limited [34]. A systematic review of the literature performed by Sawka and al. [35] selected only two papers among 172 relevant studies that specifically compared the risk of SPMs in individuals with DTC treated or not treated with radioiodine. To be included in Sawka's review, studies had to present certain quality characteristics: for example, they had to present relative risk (RR), odds ratio or hazard ratio (with respective $95 \%$ confidence intervals, CIs) data; they had to have a certain study design (cohort studies, cross-sectional studies, cancer registries, administrative datasets, or meta-analyses), and they had to include at least 50 patients.

The first selected study was conducted by pooling the DTC patients of three major European cohorts, treated at each center in the period 1934-1995 [36]. The characteristics and main results of the study are reported in Table 2.

In this study, the relationship between increasing cumulative activity of administered ${ }^{131} \mathrm{I}$ and risk of SPMs was also investigated. The authors estimated an excess relative risk (ERR) of $3.5 \%$ (95\% CI: 0.9-6.9\%) per $\mathrm{GBq}$ of ${ }^{131} \mathrm{I}$ and an excess absolute risk (EAR) of 14.4 solid cancers per GBq of administered ${ }^{131} \mathrm{I}$ and per 100,000 person-years of follow-up (PYR). The ERR for solid tumors did not vary greatly with time after exposure to ${ }^{131} \mathrm{I}$. Among the 3,211 patients followed up for at least 10 years after thyroid cancer treatment, the ERR of SPMs more than 10 years after the last ${ }^{131} \mathrm{I}$ treatment was $6 \%(95 \% \mathrm{CI}$ : $1-12 \%)$ per GBq of administered ${ }^{131} \mathrm{I}$. This study, the only one to investigate, in-depth, the role of radioiodine and to consider cumulative radioiodine activity as a time-dependent covariate, showed that RIT can cause a 10-20\% increase in SPMs and that each GBq of ${ }^{131} \mathrm{I}$ increased the risk of a second solid cancer by an average of $3.5 \%$ and of leukemia by $39 \%$.

The risk was expressed as a linear function of the cumulative activity; due to the lack of the required power, the shape of the dose-response curve could not be investigated. Considering single types of tumor, the study showed an increased risk of salivary gland, digestive tract and soft-tissue tumors and bone sarcomas linked to increasing doses of radioiodine. The first two of these sites are linked to radioiodine uptake or transit, while the latter could be exposed to high activities of radioiodine in patients treated for DTC with bone metastases. For other sites (breast, kidney and melanoma), no convincing data of increased risk due to RIT were collected; similar incidences were found in exposed versus non-exposed patients, or no dose-response relationship was demonstrated. 
Table 2 Characteristics, SIRs and RRs of the two main epidemiological studies on SPMs in patients with differentiated thyroid carcinoma submitted to radioiodine therapy

* pts. with available data on RIT and minimal latency of SPM of 36 months

\begin{tabular}{|c|c|c|}
\hline & European clinical cohorts & North American registry cohorts \\
\hline First author, year & Rubino [36] & Brown [37] \\
\hline Number of patients & 6,841 & $30,278(9,661 *)$ \\
\hline Pts treated with RIT & $4,225(62 \%)$ & $10,257(33.9 \%)$ \\
\hline Pts lost to follow-up & $20.00 \%$ & n.a. \\
\hline Age & 44 years (mean) & 42 years (median) \\
\hline Follow-up & 13 years (mean) & 8.6 years (median) \\
\hline DTC-SPM interval & 15 years (mean) & 8.1 years (median) \\
\hline SPM observed & 576 & 2,338 \\
\hline Minimal latency of SPM & 2 years & 2 months \\
\hline Overall increased risk (CI) & $27 \%(15-40)$ & $9 \%(5-14)$ \\
\hline Cancer site & SIR $(95 \% \mathrm{CI})$ of DTC patients & SIR $(95 \% \mathrm{CI})$ of DTC patients \\
\hline Bone and soft tissue & $5.9(3.6-9.0)$ & \\
\hline Endocrine glands & $3.1(1.6-5.3)$ & \\
\hline Kidney & $2.6(1.7-3.8)$ & $2.4(1.97-2.89)$ \\
\hline Skin (melanoma) & $2.5(1.6-3.7)$ & \\
\hline Central nervous system & $2.5(1.5-3.8)$ & $1.58(1.13-2.14)$ \\
\hline Oral cavity & $2.0(1.0-3.4)$ & \\
\hline Urinary tract & $1.8(1.3-2.4)$ & \\
\hline Male genital organs & $1.6(1.0-2.4)$ & \\
\hline Digestive tract & $1.3(1.0-1.5)$ & \\
\hline Female breast & $1.3(1.0-1.5)$ & $1.22(1.12-1.32)$ \\
\hline Prostate & & $1.34(1.19-1.51)$ \\
\hline Hodgkin lymphoma & & $1.75(1.0-2.85)$ \\
\hline Leukemia & & $1.41(1.1-1.79)$ \\
\hline Myeloma & & $1.63(1.16-2.22)$ \\
\hline Salivary glands & & $2.72(1.48-4.56)$ \\
\hline All sites & $1.3(1.2-1.4)$ & \\
\hline Cancer site & RR $\left({ }^{131}\right.$ I vs. no $\left.{ }^{131} \mathrm{I}\right)$ & n.a. \\
\hline Salivary glands & $7.5(1.2-143)$ & \\
\hline Bone and soft tissue & $4.0(1.5-12.4)$ & \\
\hline Female genital organs & $2.2(1.3-3.9)$ & \\
\hline Uterus & $2.3(1.2-4.7)$ & \\
\hline Leukemia & $2.5(1.0-7.4)$ & \\
\hline Overall & $1.2(1.0-1.4)$ & $1.23(1.04-1.45)$ \\
\hline
\end{tabular}

No interaction was shown between external radiotherapy and RIT, but this could be due to the small number of patients treated with both modalities. It must also be underlined that the study was not able to clarify whether the risk is increased for a single administration of $3.7 \mathrm{GBq}$ of ${ }^{131} \mathrm{I}$, or only for high cumulative activities. This is an important point, which needs to be clarified, because the effectiveness-risk ratio is particularly narrow in ablative treatments, where single low activities are administered. Conversely, in patients with distant metastases, usually treated with high cumulative activities of ${ }^{131} \mathrm{I}$ (there are no alternative treatments with fewer hazards for these patients, whose life span is shorter), a link to an increased risk is more clearly shown.
Nevertheless, it should be borne in mind that administration of repeated treatments resulting in high cumulative activities increases the risk of SPMs. Thus, repeated administrations of radioiodine should be carefully evaluated, especially in the absence of evidence of the efficacy of the treatment, such as reduction in the size of metastatic tissue, falling serum thyroglobulin levels, and improvement of symptoms or signs related to metastatic disease.

Other limitations of this study were the relatively high number of cases lost to follow-up (20\%), even though this limitation affected the exposed and non-exposed patients equally, and the scarce data in children and adolescents, known to be particularly susceptible to radiation carcinogenic effects. Finally, even though this is the only 
published study to investigate the relationship between cumulative activity of radioiodine and risk of SPMs, it must be remembered that administered activity is only one of the factors influencing biological effects and that radiation dose (not estimable in this study) would have been a better parameter to clarify the cause-effect relationship.

The second high-quality study selected in the systematic review of the literature performed by Sawka et al. [35] was conducted on a population of DTC patients drawn from 14 population-based registries of the Surveillance, Epidemiology, and End Results (SEER) program of the National Cancer Institute [37]. The characteristics and main results of this study are also reported in Table 2.

Comparing the risk of developing an SPM in patients treated (in the period 1973-2002) with radioiodine and/or external radiotherapy versus patients not treated, an overall RR of 1.16 (95\% CIs 1.05-1.27) was found. The comparison between patients treated only with radioiodine versus those not treated was possible only for the period 1988-2002. In these patients, a statistically indistinguishable RR [1.08 (95 \% CIs 0.93-1.24)] was found. However, considering a latency of 36 months as opposed to 2 months, the observed/expected ratio was found to be 1.23 (95\% CIs 1.04-1.45) in the exposed versus 1.04 (95\% CIs 0.9-1.2) in the non-exposed patients.

The strengths of this study, based on data drawn from cancer registries, were the high number of patients evaluated, the observed and expected numbers of second cancers derived from the same population, and the exclusion of possible selection or referral bias through the use of data derived directly from population-based registries. The main drawback was the lack of information about cumulative administered activity of ${ }^{131} \mathrm{I}$ and number of administrations, which precluded evaluation of the dose-effect relationship.

A recent cohort study [38], based on the SEER database, evaluated 14,589 low-risk DTC patients submitted to RIT (out of a total population of 37,176). The SIR of SPMs at any site was found to be 1.18 (95\% CI 1.10-1.25) for patients receiving RIT and 1.02 (95\% CI 0.98-1.06) for those who were not treated with RIT. The paper highlighted an increasing use of RIT in DTC patients, despite an increasing proportion of low-risk DTC, and a rising trend of SIR over time, paralleling the increasing use of RIT. Unfortunately, these observations could not be founded on data directly linked to the role of RIT in carcinogenic risk, given the lack of fundamental data in this respect, namely data on the administered activity of ${ }^{131} \mathrm{I}$, RRs and dose-response relationship.

Another study [39], also based on the SEER database, reported an RR attributable to RIT of 1.12 (95\% CI
1.01-1.25); this was obtained by evaluating 27,775 DTC patients surviving at least 5 years after first diagnosis, 10,904 of whom $(39 \%)$ were treated with ${ }^{131} \mathrm{I}$.

\section{Conclusions}

How should these data affect clinical practice?

First of all, available experimental studies, albeit of limited statistical power and quality, conclude that RIT of DTC can induce SPMs; this may be considered a predictable conclusion, both on theoretical grounds, and in view of data gathered in different settings, such as that of atomic bomb survivors [13, 35, 36].

Second, this risk is rather lower or comparable than that seen with other cancer treatment modalities, such as chemotherapy and external beam radiotherapy, which are also associated with the induction of SPMs [40]. An overall RR of induced leukemia of around 3-4 or more has been reported for chemotherapeutic drugs used to treat Hodgkin and non-Hodgkin lymphomas. External beam radiotherapy can also induce SPMs, even though the risk of leukemia is considerably smaller after radiation than after chemotherapy.

Third, it is probable that radioiodine increases the risk of leukemia and it is possible that it induces SPMs in some organs where radioiodine accumulates or transits, such as the salivary glands and gastrointestinal tract [36, 37]. Hydration of patients, use of sialagogues and laxative agents, and treatment in euthyroid state after administration of rhTSH (Thyrogen ${ }^{\circledR}$ ) are methods that can decrease exposure of such organs [4].

Fourth, it is not known whether the risk is increased only for high cumulative administered activities of radioiodine or also for single administrations of lower activities, nor has the actual risk in children and adolescents been estimated [41].

In conclusion, RIT of DTC can be defined a safe therapy, but the process of justification of treatment must be performed particularly meticulously in those cases in which the benefit of the treatment will be small or doubtful. Optimization of treatment can help to reduce exposure of non-target tissues. In this area, individualized dosimetry might make it possible to obtain, with a single administration, outcomes similar to or better than those obtained with multiple administrations of fixed activities of radioiodine, thereby decreasing both cumulative activities and actual radiation doses to critical organs [42, 43].

Conflict of interest Massimo Eugenio Dottorini and Massimo Salvatori declare that they have no conflict of interest related to the publication of this article. 
Human and Animal Studies This article does not contain any studies with human or animal subjects performed by the any of the authors.

\section{Appendix: Glossary of terms used in radiation epidemiology}

Risk

Chance of injury, loss, or detriment. A measure of the deleterious effects that may be expected as the result of an action or inaction.

\section{Epidemiology}

Study of the distribution and determinants of disease prevalence in man through which epidemiologists seek to describe the populations at risk, discover the causes of diseases, and quantify both the risk of disease and its relationship with known or suspected causal factors.

\section{Radiation epidemiology}

Epidemiological study in which the exposure to radiation is the factor of primary interest and in which epidemiologists seek to relate the risk of disease to different levels and patterns of radiation exposure. If the rates of a disease are essentially the same in the exposed and unexposed groups, there is said to be no association between radiation exposure and disease. If the disease rate is higher among those exposed to radiation, there is a positive association. If the disease rate is higher among the unexposed group, there is a negative association between radiation exposure and disease.

\section{Experimental study}

When the levels of at least one explanatory factor are under the control of the investigator, the study is said to be experimental. An example is a clinical trial designed to assess the utility of some treatment (e.g., radioiodine treatment).

\section{Observational study}

When the levels of all explanatory factors are determined by observation only, the study is observational (e.g., study of atomic bomb survivors). Most studies relevant to the evaluation of radiation risks in human populations are observational.

\section{Cohort-based/cohort study}

A study comparing disease rates among exposed and unexposed persons, in which exposure is not determined by design, is termed a "cohort", "cohort-based" or "followup" study. A cohort-based study may be performed either prospectively or retrospectively.

Case-control study

A study comparing exposure among persons with a disease of interest and persons without the disease of interest is called a case-control study.

\section{Bias}

Bias can be defined as any process at any stage of inference that tends to produce results or conclusions that differ systematically from the truth. Because epidemiological studies are observational rather than experimental in nature, bias or confounding factors may produce spurious results (e.g., one potential source of bias is the failure to obtain follow-up data for the entire population in a cohort study). Three potential forms of bias are selection bias (e.g., when enrollment into a study is dependent on both radiation exposure and disease status), information bias (e.g., when information on disease or on radiation exposure is obtained differentially from exposed or from diseased persons), and confounding bias (e.g., when a third factor exists that is related to both radiation exposure and disease effects, such as other treatments, smoking, diet, chemicals, and hereditary factors). Confounding factors may increase radiation carcinogenic risk in an additive or a multiplicative model.

\section{Standardized incidence ratio}

A standardized incidence ratio (SIR) analysis is commonly used to determine whether the occurrence of cancer in a relatively small population is higher or lower than expected. It estimates the occurrence of cancer in the cohort relative to what might be expected if the cohort population had the same cancer incidence as the normal reference population. The SIR is obtained by dividing the observed number of cases of cancer by the "expected" number of cases (i.e., the number of cases that would occur in the cohort population if the cancer rate in the normal reference population occurred in that cohort population).

\section{Confidence interval}

A confidence interval (CI) estimates the interval of an unknown parameter, such as a risk. A $95 \%$ confidence interval, for example, is constructed using a procedure that is theoretically successful in capturing the parameter of interest in $95 \%$ of its applications. Specifically, a $95 \%$ CI is the range of estimated SIR values that has a $95 \%$ 
probability of including the true SIR for the population. Confidence limits are the end points of a confidence interval. If the $95 \%$ CI does not include the unity, then the study population is significantly different from the comparison or "normal" population. If the CI is narrow, it is likely that the calculated SIR is close to the true SIR for the population; conversely, a wide interval implies that the true SIR could be much lower than or much higher than the calculated SIR.

Absolute risk and excess absolute risk

Absolute risk (AR) is the rate of disease in a population. In radiation epidemiology, the AR model assumes that the risk caused by the exposure, proportional to the dose, is independent of the baseline risk and that a specific number of excess cancers will occur as a result of exposure to ionizing radiation (e.g., 75 per 100,000 exposed persons per year).

The excess absolute risk (EAR), also termed "attributable risk" or "risk difference", is the difference between two absolute risks. It is calculated as the rate of disease in an exposed population minus the rate of disease in an unexposed population [e.g., (75 per 100,000 exposed persons per year)—(25 per 100,000 non-exposed persons per year) $=50$ per 100,000 per year]. Because the risk from the exposure is simply added to the baseline risk, the EAR model is commonly referred to as the additive model.

\section{Relative risk and excess relative risk}

In radiation epidemiology, the relative risk (RR) assumes that if a dose of radiation is administered to a population, the possible number of excess cancers will increase as the natural incidence of cancer increases with advancing age in the non-irradiated population. It is relative in the sense that it predicts a percentage increase in incidence rather than a specific number of cases. It is calculated as the ratio of the rate of disease in a group exposed to ionizing radiation divided by the rate in a group not exposed (e.g., 100 cancers per 100,000 exposed population per year/20 cancers per 100,000 non-exposed population per year $=\mathrm{RR}$ 5.0). A RR of 1 means there is no increased risk in the exposed compared with the non-exposed group, while a RR higher than 1 means that the risk is increased in the exposed group, and a RR lower than 1 indicates that the exposed group has a lower risk than the non-exposed group.

The excess relative risk (ERR) is the rate of disease in an exposed population divided by the rate of disease in an unexposed population minus 1.0 (e.g., RR 5.0-1.0 = ERR 4.0) and it is well known that for all cancer subtypes it diminishes with increasing age at exposure. In this model, the risk from the exposure is a product of both the baseline risk and the dose. For this reason, this model is often referred to as the multiplicative model.

Statistical power

Statistical power is the ability of a study to demonstrate an association or causal relationship between two variables, assuming that an association exists. For example, an $80 \%$ statistical power in a clinical trial dealing with a certain modality of treatment means that the study has an $80 \%$ chance of demonstrating that the treatment has a statistically significant effect. If the statistical power of a study is low, the study results will be questionable and it is possible that the study was too small to detect any differences. By convention, $80 \%$ is an acceptable level of statistical power.

\section{Person-years of follow-up}

Person-years of follow-up (PYR) is a measure that takes into account both the number of people in the study and the amount of time each person spends in the study. For example, a study that followed 1,000 people for 1 year would contain 1,000 person-years of data. A study that followed 100 people for 10 years would also contain 1,000 person-years of data, with the trade-off being that, in the second case, fewer people were studied, but each one for a longer period of time.

\section{References}

1. Aschebrook-Kilfoy B, Ward MH, Sabra MM, Devesa SS (2011) Thyroid cancer incidence patterns in the United States by histologic type, 1992-2006. Thyroid 21:125-134

2. Elisei R, Molinaro E, Agate L, Bottici V, Masserini L, Ceccarelli C, Lippi F, Grasso L, Basolo F, Bevilacqua G, Miccoli P, Di Coscio G, Vitti P, Pacini F, Pinchera A (2010) Are the clinical and pathological features of differentiated thyroid carcinoma really changed over the last 35 years? Study on 4,187 patients from a single Italian institution to answer this question. J Clin Endocrinol Metab 95:1516-1527

3. Mallick U, Harmer C, Yap B, Wadsley J, Clarke S, Moss L, Nicol A, Clark PM, Farnell K, McCready R, Smellie J, Franklyn JA, John R, Nutting CM, Newbold K, Lemon C, Gerrard G, AbdelHamid A, Hardman J, Macias E, Roques T, Whitaker S, Vijayan R, Alvarez P, Beare S, Forsyth S, Kadalayil L, Hackshaw A (2012) Ablation with low-dose radioiodine and thyrotropin alfa in thyroid cancer. N Engl J Med 366:1674-1685. doi:10.1056/ NEJMoa1109589

4. Luster M, Clarke SE, Dietlein M, Lassmann M, Lind P, Oyen WJ, Tennvall J, Bombardieri E (2008) European Association of Nuclear Medicine (EANM) Guidelines for radioiodine therapy of differentiated thyroid cancer. Eur J Nucl Med Mol Imaging 35:1941-1959. doi:10.1007/s00259-008-0883-1

5. Hay ID, Thompson GB, Grant CS, Bergstralh EJ, Dvorak CE, Gorman CA, Maurer MS, McIver B, Mullan BP, Oberg AL, 
Powell CC, van Heerden JA, Goellner JR (2002) Papillary thyroid carcinoma managed at the Mayo Clinic during six decades (1940-1999): temporal trends in initial therapy and long-term outcome in 2,444 consecutively treated patients. World J Surg 26:879-885

6. Sawka AM, Thephamongkhol K, Brouwers M, Thabane L, Browman G, Gerstein HC (2004) Clinical review 170: a systematic review and metaanalysis of the effectiveness of radioactive iodine remnant ablation for well-differentiated thyroid cancer. J Clin Endocrinol Metab 89:3668-3676

7. American Thyroid Association (ATA) Guidelines Taskforce on Thyroid Nodules and Differentiated Thyroid Cancer, Cooper DS, Doherty GM, Haugen BR, Kloos RT, Lee SL, Mandel SJ, Mazzaferri EL, McIver B, Pacini F, Schlumberger M, Sherman SI, Steward DL, Tuttle RM. (2009). Revised American Thyroid Association management guidelines for patients with thyroid nodules and differentiated thyroid cancer. Thyroid. 19:1167-214. doi:10.1089/thy.2009.0110. Erratum in: Thyroid. (2010) 20:674-5. Thyroid. (2010) 20:942. Hauger, Bryan R [corrected to Haugen, Bryan R]

8. Little MP (2003) Risks associated with ionizing radiation. Br Med Bull 68:259-275

9. Van Nostrand D (2009) The benefits and risks of I-131 therapy in patients with well-differentiated thyroid cancer. Thyroid 19:1381-1391. doi:10.1089/thy.2009.1611

10. Effects of ionizing radiation: United Nations Scientific Committee on the Effects of Atomic Radiation-UNSCEAR 2000 Report, vol II-Report to the General Assembly, with Scientific Annexes F, G, H, I and J. vol II: Effects

11. Effects of ionizing radiation: United Nations Scientific Committee on the Effects of Atomic Radiation-UNSCEAR 2001 Report-Report to the general assembly, with Scientific Annex: Hereditary Effects of Radiation

12. NCRP (National Council on Radiation Protection and Measurement) Report No. 136 Evaluation of the linear-nonthreshold doseresponse model for ionizing radiation. Bethesda, MD, National Council on Radiation Protection and Measurement

13. Health risks from exposure to low levels of ionizing radiation. BEIR VII PHASE 2. Committee to assess health risks from exposure to low levels of ionizing radiation. Board on radiation effects research. Division on earth and life studies. National research council of the national academies. The National Academies Press, Washington, DC. www.nap.edu

14. Hendee WR, O'Connor MK (2012) Radiation risks of medical imaging: separating fact from fantasy. Radiology 264:312-321. doi:10.1148/radiol.12112678

15. Brenner DJ, Doll R, Goodhead DT, Hall EJ, Land CE, Little JB, Lubin JH, Preston DL, Preston RJ, Puskin JS, Ron E, Sachs RK, Samet JM, Setlow RB, Zaider M (2003) Cancer risks attributable to low doses of ionizing radiation: assessing what we really know. Proc Natl Acad Sci USA 100:13761-13766

16. Royal HD (2008) Effects of low level radiation-what's new? Semin Nucl Med 38:392-402. doi:10.1053/j.semnuclmed.2008. 05.006

17. Hanahan D, Weinberg RA (2011) Hallmarks of cancer: the next generation. Cell 144:646-674. doi:10.1016/j.cell.2011.02.013. Review

18. Kim IG, Lee YS (2003) Radiation-induced tumorigenesis. J Biochem Mol Biol 36:144-148

19. Little JB (2003) Genomic instability and radiation. J Radiol Prot 23:173-181

20. Kolbert KS, Pentlow KS, Pearson JR, Sheikh A, Finn RD, Humm JL, Larson SM (2007) Prediction of absorbed dose to normal organs in thyroid cancer patients treated with ${ }^{131} \mathrm{I}$ by use of $124 \mathrm{I}$ PET and 3-dimensional internal dosimetry software. J Nucl Med 48:143-149
21. Effects of ionizing radiation: United Nations Scientific Committee on the Effects of Atomic Radiation-UNSCEAR 1994 Report-Report to the general assembly, with Scientific Annexes $A$ and $B$ : sources and effects of ionizing radiation

22. Effects of ionizing radiation: United Nations Scientific Committee on the Effects of Atomic Radiation-UNSCEAR 2000 Report, vol II-Report to the general assembly, with scientific annexes F, G, H, I and J. vol II Annex I: Epidemiological evaluation of radiation-induced cancer

23. Salvatori M, Cremonesi M, Indovina L, Chianelli M, McEwan AJB, and Zanzonico P (2012) Radiobiology and radiation dosimetry in nuclear medicine: therapy, diagnosis, and considerations for sensitive populations in nuclear oncology-pathophysiology and clinical applications. In: Strauss HW, Mariani G, Volterrani D, Larson SM (eds) doi:10.1007/978-0-387-48894-3 Springer, New York

24. Lassmann M, Hänscheid H, Gassen D, Biko J, Meineke V, Reiners C, Scherthan H (2010) In vivo formation of gamma-H2AX and 53BP1 DNA repair foci in blood cells after radioiodine therapy of differentiated thyroid cancer. $J$ Nucl Med 51:1318-1325. doi:10.2967/jnumed.109.071357

25. Federico G, Boni G, Fabiani B, Fiore L, Lazzeri P, Massart F, Traino C, Verola C, Saggese G, Mariani G, Scarpato R (2008) No evidence of chromosome damage in children and adolescents with differentiated thyroid carcinoma after receiving ${ }^{131} \mathrm{I}$ radiometabolic therapy, as evaluated by micronucleus assay and microarray analysis. Eur $\mathrm{J}$ Nucl Med Mol Imaging 35:2113-2121. doi:10.1007/s00259-008-0867-1

26. Mariotto AB, Rowland JH, Ries LA, Scoppa S, Feuer EJ (2007) Multiple cancer prevalence: a growing challenge in long-term survivorship. Cancer Epidemiol Biomarkers Prev 16:566-571

27. Subramanian S, Goldstein DP, Parlea L, Thabane L, Ezzat S, Ibrahim-Zada I, Straus S, Brierley JD, Tsang RW, Gafni A, Rotstein L, Sawka AM (2007) Second primary malignancy risk in thyroid cancer survivors: a systematic review and meta-analysis. Thyroid 17:1277-1288

28. Ronckers CM, McCarron P, Engels EA, Ron E, New malignancies following cancer of the thyroid and other endocrine glandsnew malignancies among cancer survivors: SEER Cancer Registries 1973-2000 (2006) In: Curtis RE, Freedman DM, Ron E, Ries LAG, Hacker DG, Edwards BK,Tucker MA,Fraumeni JF Jr (eds) National Cancer Institute, NIH Publ. No. 05-5302. Bethesda, MD

29. Links TP, van Tol KM, Meerman GJ, de Vries EG (2001) Differentiated thyroid carcinoma: a polygenic disease. Thyroid 11:1135-1140

30. Lupoli G, Vitale G, Caraglia M, Fittipaldi MR, Abbruzzese A, Tagliaferri P, Bianco AR (1999) Familial papillary thyroid microcarcinoma: a new clinical entity. Lancet 353:637-639

31. Lloyd KM, Dennis M (1963) Cowden's disease: a possible new symptom complex with multiple system involvement. Ann Intern Med 58:136-142

32. Lal G, Gallinger S (2000) Familial adenomatous polyposis. Semin Surg Oncol 18:314-323

33. Sandeep TC, Strachan MWJ, Reynolds RM, Brewster DH, Sce'lo G, Pukkala E, Hemminki K, Anderson A, Tracey E, Friis S, McBride ML ML et al (2006) Second primary cancers in thyroid cancer patients: a multinational record linkage study. J Clin Endocrinol Metab 91:1819-1825

34. Ron E (2008) Treatment for thyroid cancer as a risk factor for a second malignancy. Available at: http://www.thyroid.org/ wpcontent/uploads/publications/clinthy/clinthy_v202.pdf Clin Thyroidol 20:3-4.

35. Sawka AM, Thabane L, Parlea L, Ibrahim-Zada I, Tsang RW, Brierley JD, Straus S, Ezzat S, Goldstein DP (2009) Second primary malignancy risk after radioactive iodine treatment for 
thyroid cancer: a systematic review and meta-analysis. Thyroid 19:451-457. doi:10.1089/thy.2008.0392

36. Rubino C, de Vathaire F, Dottorini ME, Hall P, Schvartz C, Couette JE, Dondon MG, Abbas MT, Langlois C, Schlumberger M (2003) Second primary malignancies in thyroid cancer patients. Br J Cancer 89:1638-1644

37. Brown AP, Chen J, Hitchcock YJ, Szabo A, Shrieve DC, Tward JD (2008) The risk of second primary malignancies up to three decades after the treatment of differentiated thyroid cancer. J Clin Endocrinol Metab 93:504-515

38. Iyer NG, Morris LG, Tuttle RM, Shaha AR, Ganly I (2011) Rising incidence of second cancers in patients with low-risk (T1N0) thyroid cancer who receive radioactive iodine therapy. Cancer 117:4439-4446. doi:10.1002/cncr.26070

39. Berrington de Gonzalez A, Curtis RE, Kry SF, Gilbert E, Lamart S, Berg CD, Stovall M, Ron E (2011) Proportion of second cancers attributable to radiotherapy treatment in adults: a cohort study in the US SEER cancer registries. Lancet Oncol 12:353-360. doi:10.1016/S1470-2045(11)70061-4

40. Travis LB (2006) The epidemiology of second primary cancers. Cancer Epidemiol Biomarkers Prev 15:2020-2026

41. de Vathaire F (2008) The carcinogenic effects of radioiodine therapy for thyroid carcinoma. Nat Clin Pract Endocrinol Metab 4:180-181. doi:10.1038/ncpendmet0761

42. Klubo-Gwiezdzinska J, Van Nostrand D, Atkins F, Burman K, Jonklaas J, Mete M, Wartofsky L (2011) Efficacy of dosimetric versus empiric prescribed activity of ${ }^{131} \mathrm{I}$ for therapy of differentiated thyroid cancer. J Clin Endocrinol Metab 96:3217-3225. doi:10.1210/jc.2011-0494

43. Chiesa C, Castellani MR, Vellani C, Orunesu E, Negri A, Azzeroni R, Botta F, Maccauro M, Aliberti G, Seregni E, Lassmann M, Bombardieri E (2009) Individualized dosimetry in the management of metastatic differentiated thyroid cancer. Q J Nucl Med Mol Imaging 53:546-561 